\title{
A Testbed for MANETs: Implementation, Experiences and Learned Lessons
}

\author{
Leonard Barolli, Member, IEEE, Makoto Ikeda, Member, IEEE, Fatos Xhafa, Member, IEEE, and \\ Arjan Duresi, Senior Member, IEEE
}

\begin{abstract}
In this paper, we present the implementation, experiences and lessons learned of our tesbed for Ad-hoc networks and Mobile Ad hoc Networks (MANETs). We used OLSR protocol for real experimental evaluation. We investigate the effect of mobility and topology changing in the throughput of a MANET. We study the impact of best-effort traffic for Mesh Topology and Linear Topology. In this work, we consider eight experimental models and we assess the performance of our testbed in terms of throughput, round trip time and packet loss. We found that some of the OLSR's problems can be solved, for instance the routing loop, but this protocol still has the self-interference problem. Also, there is an intricate interdependence between MAC layer and routing layer. We carried out the experiments considering stationary nodes of an Ad-hoc network and the node mobility of MANETs. We found that throughput of TCP was improved by reducing Link Quality Window Size (LQWS). For TCP data flow, we got better results when the LQWS value was 10 . Moreover, we found that the node join and leave operations increase the packet loss. The OLSR protocol has a good performance when the source node is moving. However, the performance is not good when the relay nodes are moving.
\end{abstract}

Index Terms-MANET testbed, network protocols, node mobility, OLSR, performance analysis.

\section{INTRODUCTION}

$\mathbf{T}$ HE wireless mobile networks and devices are becoming increasingly popular and they provide users access to information and communication anytime and anywhere. The conventional wireless networks are often connected to a wired network. This kind of wireless network requires a fixed wireline backbone infrastructure. All mobile hosts in a communication cell can reach a base station on the wireline networks in one-hop radio transmission. In contrast, the class of Mobile Ad Hoc Networks (MANETs) is a collection of wireless mobile terminals that are able to dynamically form a temporary network without any aid from fixed infrastructure or centralized administration.

Manuscript received September 14, 2009. Date of publication May 03, 2010; date of current version June 03, 2010. This work was supported by a Grant-in-Aid for scientific research of the Japan Society for the Promotion of Science (JSPS)

L. Barolli is with the Department of Information and Communication Engineering, Fukuoka Institute of Technology (FIT), Fukuoka 811-0295, Japan (e-mail: barolli@fit.ac.jp).

M. Ikeda is with the Center for Asian and Pacific Studies, Seikei University, Tokyo 180-8633, Japan (e-mail: makoto.ikd@acm.org).

F. Xhafa is with Department of Languages and Informatics Systems Technical University of Catalonia, 08034 Barcelona, Spain (e-mail: fatos@1si.upc.edu).

A. Durresi is with Department of Computer and Information Science, Indiana University-Purdue University Indianapolis (IUPUI), Indianapolis, IN 46202 USA (e-mail: durresi@cs.iupui.edu).

Color versions of one or more of the figures in this paper are available online at http://ieeexplore.ieee.org.

Digital Object Identifier 10.1109/JSYST.2010.2047174
The nodes of MANET intercommunicate through single-hop and multihop paths in a peer-to-peer fashion. Intermediate nodes between two pairs of communication nodes act as routers. Thus the nodes operate both as hosts and routers. The nodes are mobile, so the creation of routing paths is affected by the addition and deletion of nodes. The topology of the network may change rapidly and unexpectedly.

The Ad-hoc networks and MANETs are useful in many applications environments such as collaborative computing and communications in smaller areas (building organizations, conferences, and so on). Communications in battlefields and disaster recovery areas are other examples of application environments. Similarly, communications using a network of sensors or using floats over water are other potential applications. The increase use of collaborative applications and wireless devices may further add to the needs and usage of Ad-hoc networks and MANETs. It should be noted that mobility and the absence of any fixed infrastructure make MANET very attractive for mobility and rescue operations and time-critical applications.

In general, the research for Ad-hoc networks and MANETs is focused on specific problems of the networking stack, by trying to emphasize the causes of performance degradation. Most of the work has been done in simulation, as general purpose simulators can furnish a quick and inexpensive understanding of protocols and algorithms. Simulation is indeed unavoidable to analyze the scaling behavior of ad-hoc networks which can count more than hundred nodes, and also because an exhaustive mathematical model is often too complicated. Few results are known only in simplified scenarios. However, experiments in the realworld are fundamentals in order to verify the simulation results and, if necessary, to revise the models implemented in the simulators. A typical example of this approach has revealed many aspects of IEEE 802.11, like the gray-zones effect [1], which usually are not taken into account in standard simulators, as the well-known $n s-2$ simulator.

So far we can count a lot of simulation results on the performance of Ad-hoc networks and MANETs, e.g., in terms of end-to-end throughput, delay and packet loss. However, in order to assess the simulation results, real-world experiments are needed and a lot of testbeds have been built to date [2]. The baseline criteria usually used in real-world experiments is guaranteeing the repeatability of tests, i.e., if the system does not change along the experiments. How to define a change the parameters in the system is not a trivial problem in Ad-hoc networks and MANETs, especially if the nodes are mobile.

In this paper, we concentrate on the implementation and performance analysis of a small testbed for Ad-hoc networks and MANETs. We use Optimized Link State Routing (OLSR) protocol, which is a proactive routing protocol, and it has been 


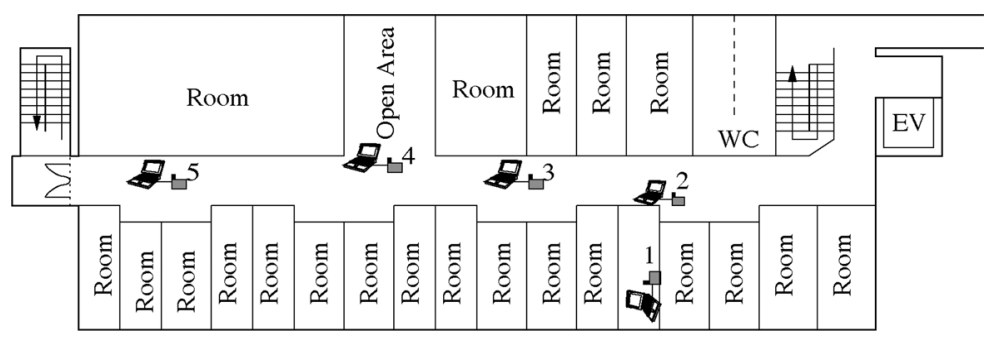

Fig. 1. STA model.

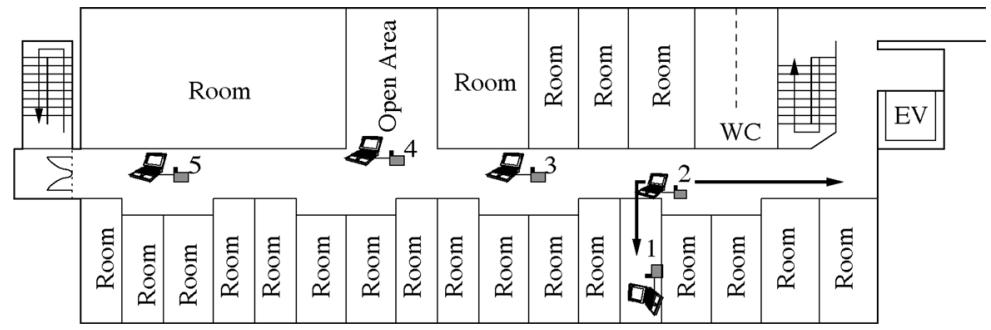

Fig. 2. MV1 model.

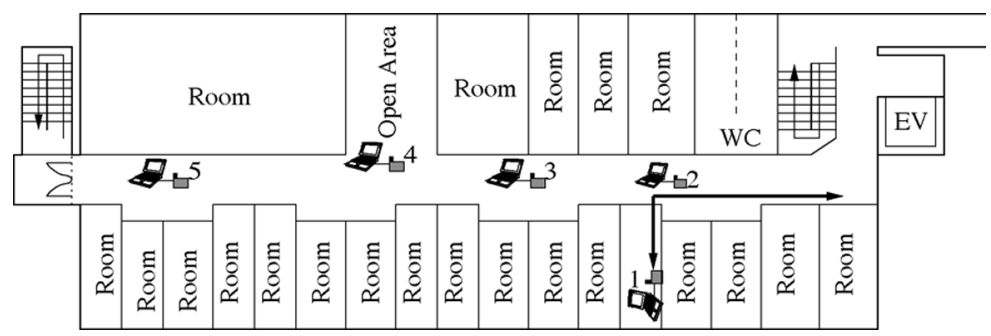

Fig. 3. MV2 model.

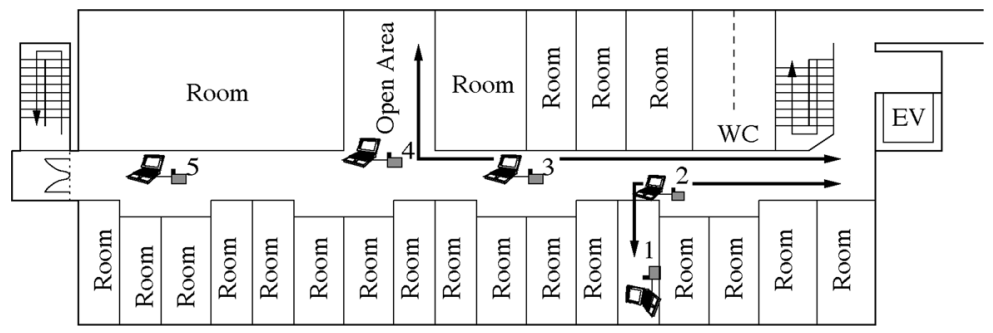

Fig. 4. MV3 model.

gaining great attention within the scientific community. Furthermore, the olsrd [3] software we have used in our experiments is the most updated software we have encountered.

In this work, we investigate the topology changing of MANET. We implemented 8 experimental models and carried out experiments for different topologies.

The structure of the paper is as follows. In Section II, we present the related work. In Section III, we present the testbed design and implementation. In Section IV, we present experimental evaluation. Finally, conclusions are given in Section V.

\section{RELATED WORK}

In [4], the authors analyze the performance of an outdoor ad-hoc network, but their study is limited to reactive protocols such as Ad hoc On Demand Distance Vector (AODV) and Dynamic Source Routing (DSR). The authors of [5], performs outdoor experiments of non standard proactive protocols. Other ad-hoc experiments are limited to identify MAC problems, by providing insights on the one-hop MAC dynamics as shown in [6].
The closest work to ours is that in [7]. However, the authors did not care about the routing protocol. In [8], the disadvantage of using hysteresis routing metric is presented through simulation and indoor measurements. Our experiments are concerned with the interaction of transport protocols and routing protocol, for instance OLSR. Furthermore, we compare the performance of the testbed for eight scenarios.

In [9], the authors present an experimental comparison of OLSR using the standard hysteresis routing metric and the Expected Transmission Count (ETX) metric in a $7 \times 7$ grid of closely spaced Wi-Fi nodes to obtain more realistic results. The throughput results are similar to our previous work and are effected by hop distance [10].

\section{Testbed Design AND IMPLEMENTATION}

\section{A. Target Environment of MANET}

We have implemented a MANET testbed which provides a realistic platform for analysing various aspect of these networks, 


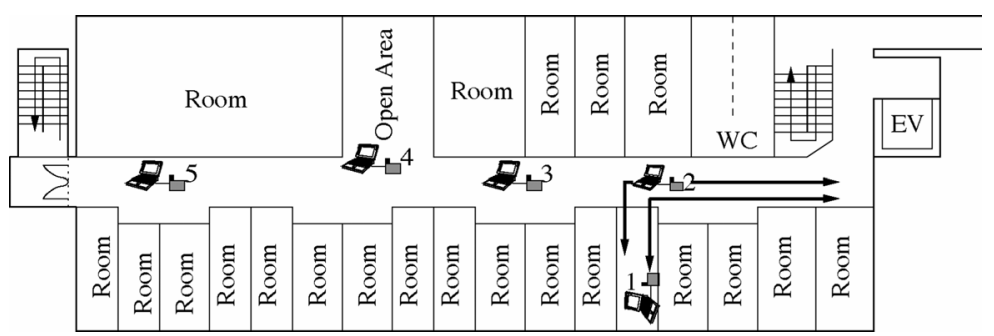

Fig. 5. MV4 model.

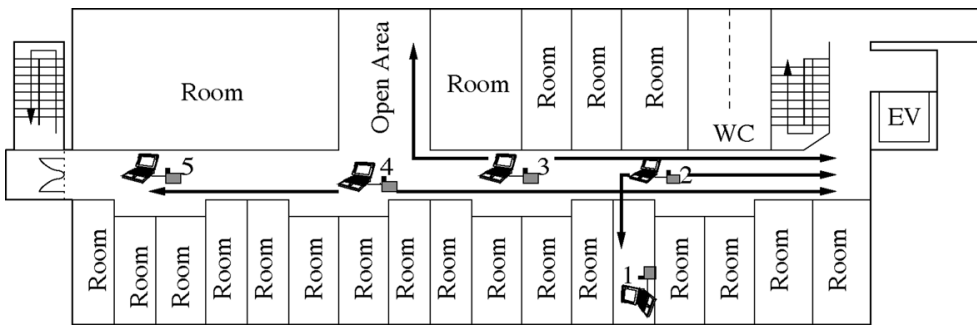

Fig. 6. MV5 model.

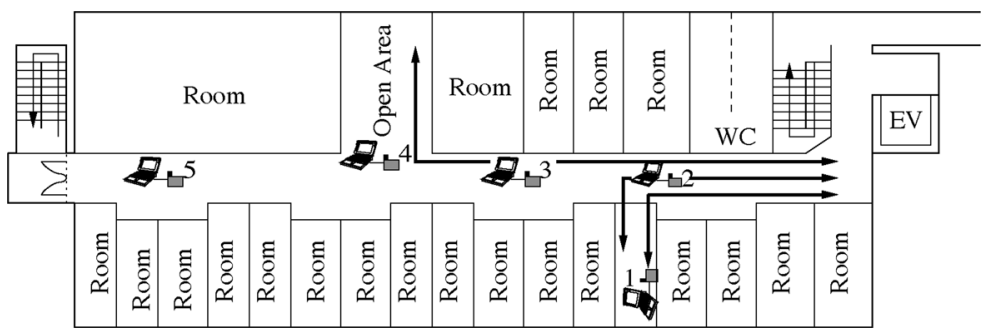

Fig. 7. MV6 model.

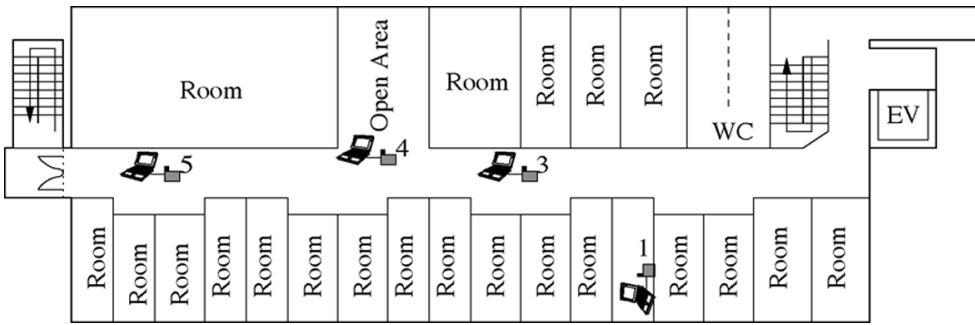

Fig. 8. MV7 model.

TABLE I

NUMBER OF ClASSIFY NODES OF EACH EXPERIMENTAL MODELS

\begin{tabular}{l|c|c|c}
\hline \hline Model & \multicolumn{2}{|c|}{ Number of moving nodes } & Num. of stati- \\
\cline { 2 - 3 } & Source node & Relay node & onary nodes \\
\hline \hline STA & 0 & 0 & 5 \\
MV1 & 0 & 1 & 4 \\
MV2 & 1 & 0 & 4 \\
MV3 & 0 & 2 & 3 \\
MV4 & 1 & 1 & 3 \\
MV5 & 0 & 3 & 2 \\
MV6 & 1 & 2 & 2 \\
MV7 & 0 & 1 & 4 \\
\hline
\end{tabular}

including the different topology models. For our testbed, we make the following considerations.

- We consider an indoor environment at our departmental floor.

- We constructed 8 experimental models: Static Model (Ad-hoc network model, where all nodes are in stationary state); Model 1 (only one relay node is moving); Model 2 (only one source node is moving); Model 3 (two relay nodes are moving); Model 4 (one relay node and one source node are moving); Model 5 (all relay nodes are moving); Model 6 (one source node and two relay node are moving) and Model 7 (Mobile node 2 is hidden between 50 and $100 \mathrm{~s}$ ).

- The mobile nodes move toward the destination at a regular speed. When the mobile nodes arrive at the corner, they stop for about $3 \mathrm{~s}$.

- In order to make the experiments easier, we implemented a testbed interface and web tool.

- Experimental time is $150 \mathrm{~s}$.

\section{B. OLSR Overview}

The link state routing protocol that is most popular today in the open source world is OLSR from olsr.org. OLSR with Link Quality (LQ) extension and fisheye-algorithm works quite well. The OLSR protocol is a pro-active routing protocol, which builds up a route for data transmission by maintaining a routing table inside every node of the network. The routing table is computed upon the knowledge of topology information, which 
is exchanged by means of Topology Control (TC) packets. OLSR makes use of HELLO messages to find its one hop neighbors and its two hop neighbors through their responses. The sender can then select its Multi Point Relays (MPR) based on the one hop node which offer the best routes to the two hop nodes. By this way, the amount of control traffic can be reduced. Each node has also an MPR selector set which enumerates nodes that have selected it as an MPR node. OLSR uses TC messages along with MPR forwarding to disseminate neighbor information throughout the network. Host Network Address (HNA) messages are used by OLSR to disseminate network route advertisements in the same way TC messages advertise host routes.

OLSRv2 is currently being developed at IETF. It maintains many of the key features of the original protocol including MPR selection and dissemination. Key differences are the flexibility and modular design using shared components such as packet format packetbb and neighborhood discovery protocol.

In our OLSR code, a simple RFC-compliant heuristic is used [11] to compute the MPR nodes. Every node computes the path towards a destination by means of a simple shortest-path algorithm, with hop-count as target metric. In this way, a shortest path can result to be also not good, from the point of view of the packet error rate. Accordingly, recently olsrd has been equipped with the LQ extension, which is a shortest-path algorithm with the average of the packet error rate as metric. This metric is commonly called as the ETX, which is defined as $\operatorname{ETX}(i)=1 /(N I(i) \times L Q I(i))$. Given a sampling window $W, N I(i)$ is the packet arrival rate seen by a node on the $i$-th link during $W$. Similarly, $\mathrm{LQI}(i)$ is the estimation of the packet arrival rate seen by the neighbor node which uses the $i$-th link. When the link has a low packet error rate, the ETX metric is higher. The LQ extension greatly enhances the packet delivery ratio with respect to the hysteresis-based technique [12].

\section{Testbed Description}

Our testbed is composed of five laptops. We constructed 8 experimental models as shown in Figs. 1-8. The experimental parameters are shown in Table I. In Fig. 1, all nodes are in a stationary state. We call this model STA. In Fig. 2, only one relay node (node 2 ) is moving. We call this model MV1. In the second model only one source node (node 1) is moving (see Fig. 3). The mobile node moves toward the destination at a regular speed. When the mobile node arrives at the corner, it stops for about $3 \mathrm{~s}$. The round-trip time is $50 \mathrm{~s}$. We call this model MV2. In the third model, two relay nodes are moving (node 2 and node 3 ) as shown in Fig. 4. We call this model MV3. In the forth model, source node and one relay node (node 2) are moving as shown in Fig. 5. We call this model MV4. In Fig. 6, all relay nodes (node 2, node 3 and node 4) are moving. We call this model MV5. In Fig. 7, source node and two relay node are moving. We call this model MV6 ${ }^{1}$. In Fig. 8, mobile node 2 is hidden between 50 and $100 \mathrm{~s}$. The round-trip time of node 3 is about $80 \mathrm{~s}$. We call this model MV7.

The operating system mounted on these machines is Fedora Core 4 Linux with kernel 2.6.x, suitably modified in order to support the wireless cards. The wireless network cards are from

${ }^{1}$ In MV2, MV4 and MV6, the source node is always moving.
Linksys. They are usb-based cards with and external antenna of $2 \mathrm{dBi}$ gain, transmitted power of $16 \pm 1 \mathrm{dBm}$ and receive sensitivity of $-80 \mathrm{dBm}$. We verified that the external antenna improves the quality of the first hop link, which is the link connecting the ad-hoc network. The driver can be downloaded from the web site in [13].

The source node serves as HTTP, FTP and DNS for the nodes in the testbed. These features are provided by the iptables mechanism, readily available under Linux machine. The source node used to coordinates the measurement campaign, as well as graphical tools to check network connectivity.

In our testbed, we have two systematic background or interference traffic we could not eliminate: the control traffic and the other wireless APs interspersed within the campus. The control traffic is due to the ssh program, which is used to remotely start and control the measurement software on the source node. The other traffic is a kind of interference, which is typical in an academic scenario.

\section{Testbed Interface}

Until now, all the parameters settings and editing were done by using command lines of bash shell (terminal), which resulted in many misprints and the experiments were repeated many times. In order to make the experiments easier, we implemented a testbed interface. For the Graphical User Interface (GUI) we used wxWidgets tool and each operation is implemented by Perl language. wxWidgets is a cross-platform GUI and tools library for GTK, MS Windows and Mac OS.

We implemented many parameters in the interface such as transmission duration, number of trials, source address, destination address, packet rate, packet size, LQWS, and topology setting function. We can save the data for these parameters in a text file and can manage in a better way the experimental conditions. Moreover, we implemented collection function of experimental data in order to make easier the experimenter's work.

\section{EXPERIMENTAL RESULTS}

\section{A. Experimental Settings}

The experimental parameters are shown in Table II. We study the impact of best-effort traffic for Mesh Topology (MT) and Linear Topology (LT). In the MT scheme, the MAC filtering routines are not enabled. We collected data for three metrics: the throughput, Round-Trip Time (RTT) and packet loss. These data are collected by using the Distributed Internet Traffic Generator (D-ITG) [14], which is an open-source Internet traffic generator. D-ITG computes the packet loss as the number of lost packet divided by the effective number of sent packets.

In previous experiments [15], [10], [16], we realized that an external antenna improves radio signal reception. The transmission rate of the data flows is $122 \mathrm{pps}=499.712 \mathrm{Kbps}$, i.e., the packet size of the payload is 512 bytes. All experiments have been performed in indoor environment, within our departmental floor of size roughly $100 \mathrm{~m}$. All laptops are in radio range of each other. In our previous work, one experiment lasted about $10 \mathrm{~s}$ and was repeated 50 times. But, the experimental time was very short. For this reason, in this paper we set the experimental time about $150 \mathrm{~s}$. We measured the throughput for TCP and 


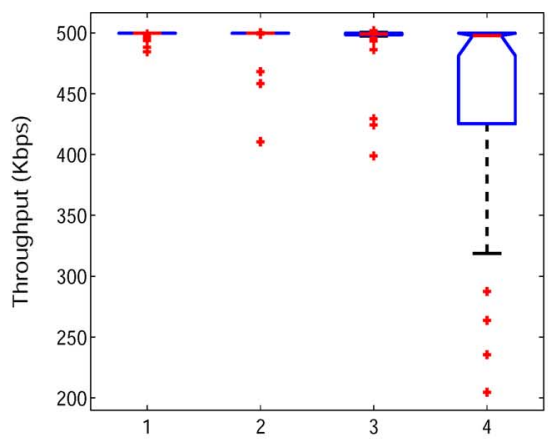

(a)

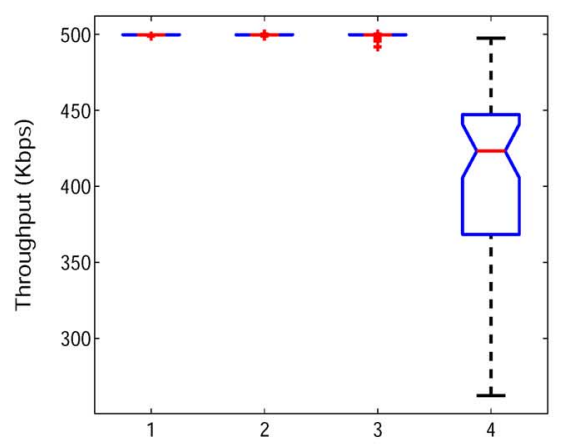

(d)

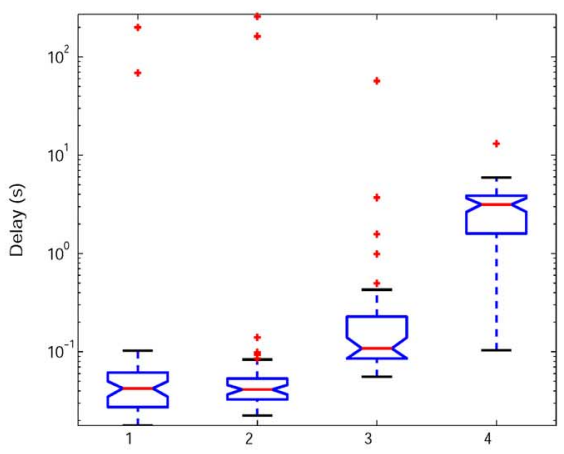

(b)

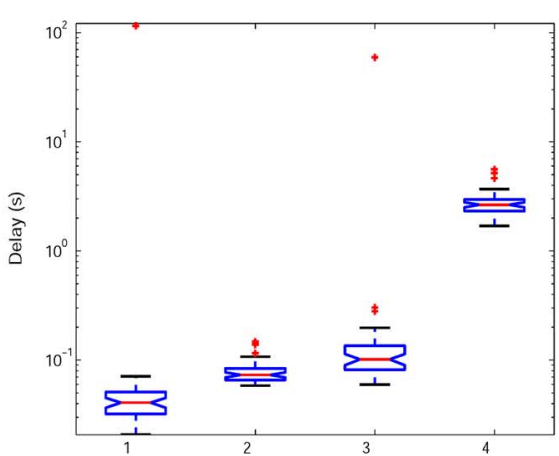

(e)

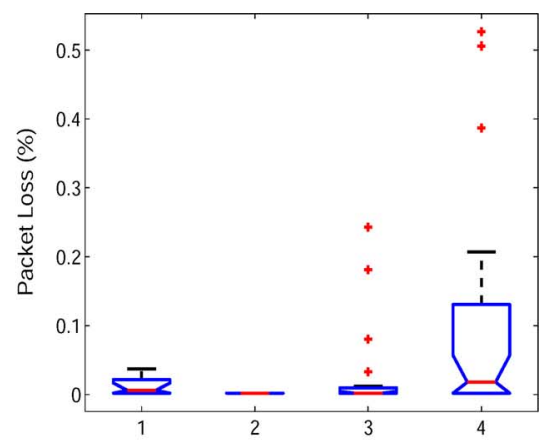

(c)

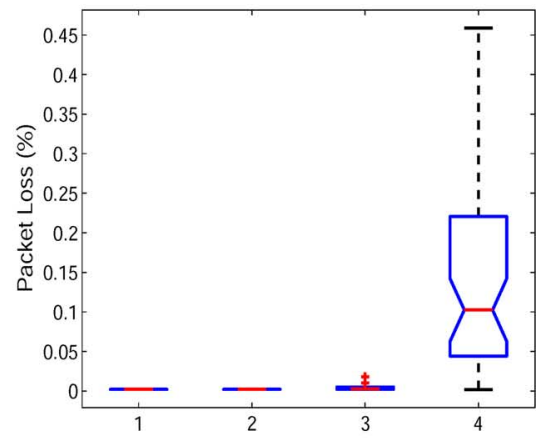

(f)

Fig. 9. Results for Ad hoc Network. (a) Goodput, MT; (b) RTT, MT; (c) Loss, MT; (d) Goodput, LT; (e) RTT, LT; (f) Packet Loss, LT.

TABLE II

EXPERIMENTAL PARAMETERS

\begin{tabular}{c||c}
\hline Function & Value \\
\hline \hline Number of Nodes & 5 \\
MAC & IEEE 802.11 \\
Packet Rate & $122 \mathrm{pps}$ \\
Number of Trials & 50,5 \\
Packet Size & $512 \mathrm{bytes}$ \\
Duration & $10000,150000 \mathrm{msec}$ \\
Protocol & OLSR \\
LQ Window Size & 10 \\
\hline
\end{tabular}

UDP, which is computed at the receiver. We estimate the packet loss to compute the link quality metrics, e.g., LQ. For OLSR, $w T_{\mathrm{HELLO}}<T_{\mathrm{Exp}}$, where $T_{\mathrm{Exp}}$ is the total duration of the experiment, i.e., in our case, $T_{\mathrm{Exp}}=150 \mathrm{~s}$, and $T_{\text {HELLO }}$ is the rate of the HELLO messages. However, the testbed was turned on even in the absence of measurement traffic. Therefore, the effective $T_{\text {Exp }}$ was much greater.

As MAC protocol, we used IEEE 802.11b. The transmission power was set in order to guarantee a coverage radius equal to the maximum allowed geographical distance in the network. Since we were interested mainly in the performance of the routing protocol, we kept unchanged all MAC parameters, such as the carrier sense, the retransmission counter, the contention window and the RTS/CTS threshold. Moreover, the channel central frequency was set to $2.412 \mathrm{GHz}$ (channel 1). In regard to the interference, it is worth noting that, during our tests, almost all the IEEE 802.11 spectrum had been used by other access points disseminated within the campus. In general, the interference from other access points is a non-controllable parameter.

\section{B. Experimental Measurements and Evaluation}

1) Evaluation of Topology Types: In order to show the range of variability of the data, we also report the box plot of the metrics as shown in Fig. 9. The box plot shows the medians, the lower and the upper quartile (the 25th and 75th percentile, respectively) and the outliers. The ends of the whiskers can represent the lowest datum which is still within 1.5 interquartile range of the lower quartile, and the highest datum which is still within 1.5 interquartile range of the upper quartile.

As shown in Fig. 9 and in Table III, we consider two topologies: LT and MT. From the point of view of OLSR operations, the LT topology is the most fragile topology, because the degree of every node is 2 . If one link becomes asymmetric, OLSR will not use it, and the node becomes isolated along with a disconnection of the entire network. By studying the LT, we can observe the impact of fluctuations of radio links quality on the selection of the MPRs, and thus on the responsiveness of the protocol. The LT is realized by means of MAC filtering. Although a self-interference remains in the LT, this way is much more practical than masking the wireless cards with copper-tapes. In the MT, the MAC filtering routines are not enabled.

The throughput drops rapidly after the third hop. Let us note that this happens for both MT and LT cases. It seems that the topology could not exploit direct links. In this case, the hop count threshold can be due to some hop in the network which is suffering by impairments of the radio links and/or MAC problems, as the gray-zones problem. Due to the fixed sampling window of the link quality sensing mechanism, nodes use routes with low quality. Consequently, a dynamic adaptation of the neighbors sensing messages rate could ameliorate the situation. This behavior is confirmed also by measurements of packet loss 


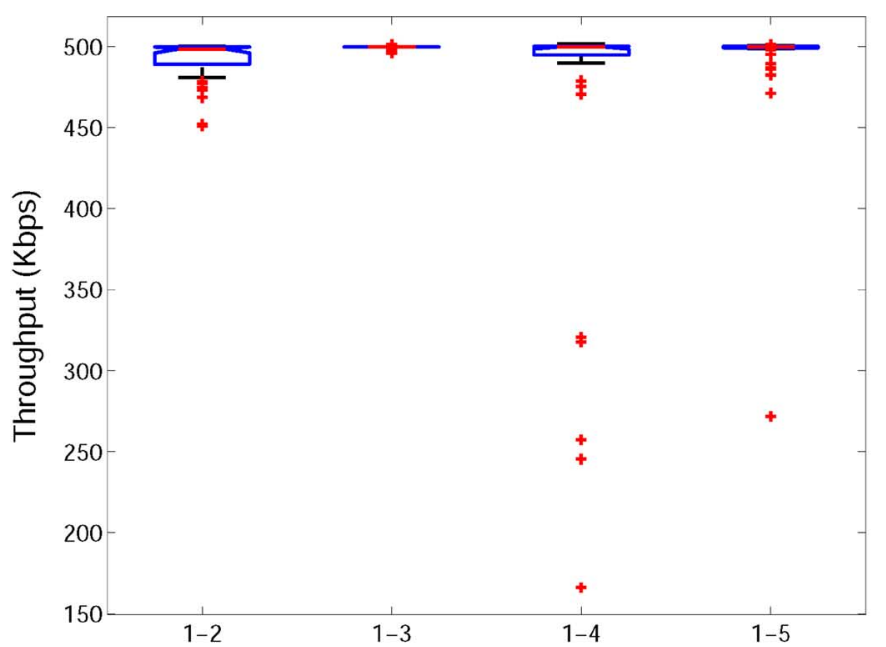

(a)

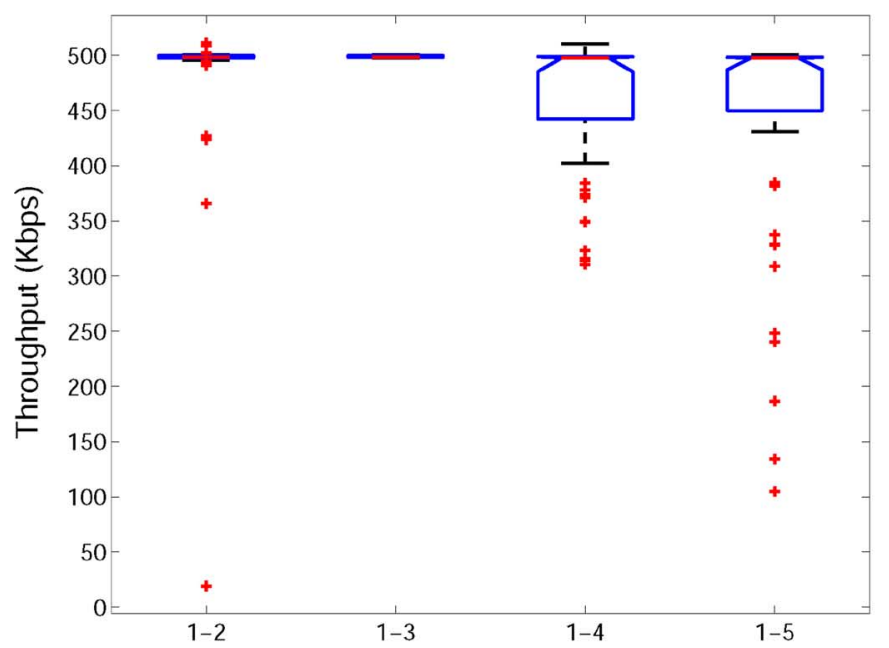

(c)

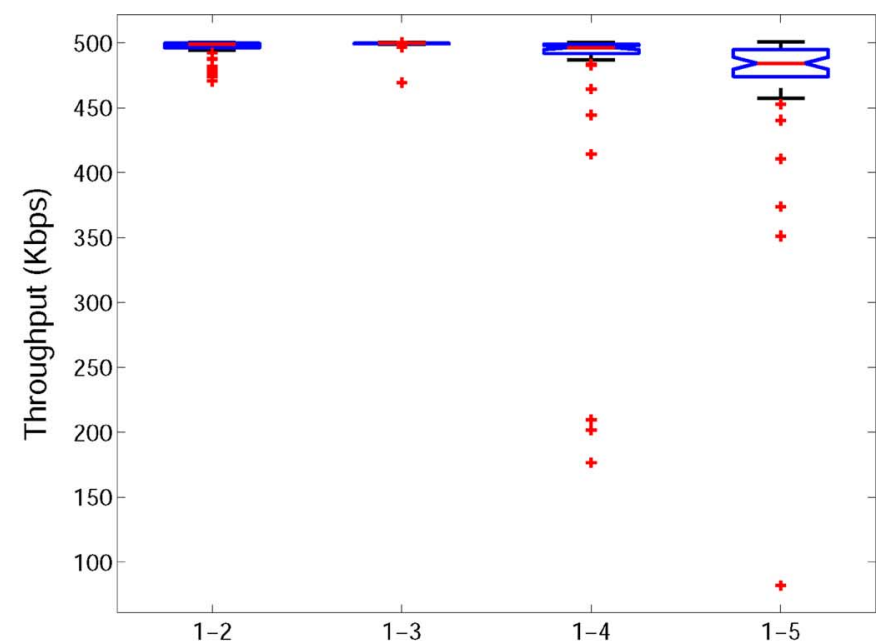

(b)

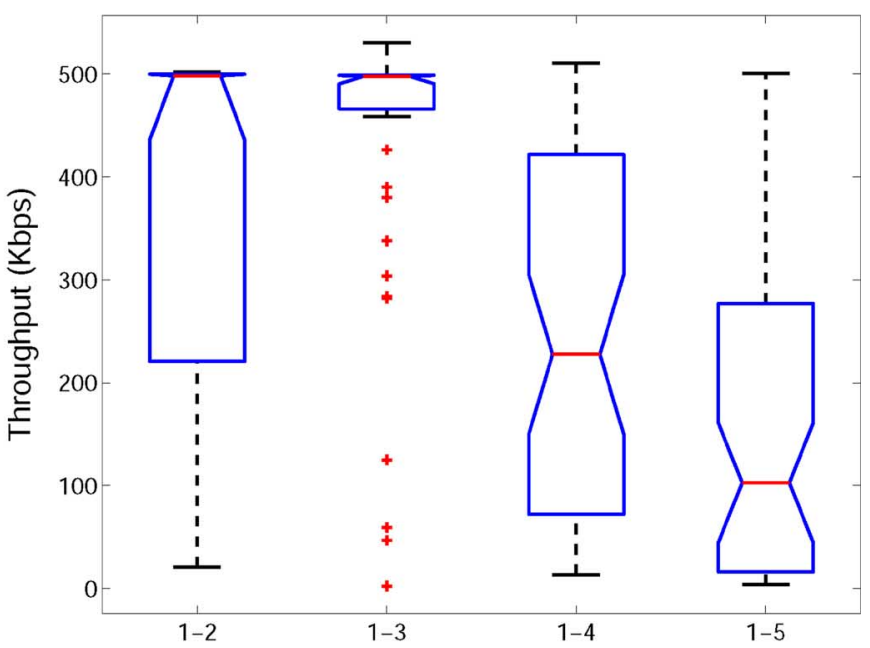

(d)

Fig. 10. Throughput results for MV1. (a) UDP, LQWS10; (b) UDP, LQWS100; (c) TCP, LQWS10; (d) TCP, LQWS100.

TABLE III

Results of Median VALues (GoOdPut, RTT, PACKet Loss)

\begin{tabular}{ccc}
\hline Model & $1(1 \rightarrow 2)$ & $2(1 \rightarrow 3)$ \\
\hline \hline STA, Mesh & $(499.712,0.042,0.006)$ & $(499.712,0.041,0.002)$ \\
STA, Linear & $(499.712,0.041,0.002)$ & $(499.712,0.070,0.002)$ \\
& $3(1 \rightarrow 4)$ & $4(1 \rightarrow 5)$ \\
\hline \hline & $(499.213,0.108,0.002)$ & $(497.824,3.152,0.017)$ \\
& $(499.712,0.101,0.003)$ & $(442.174,2.647,0.102)$ \\
\hline
\end{tabular}

and RTT. In this case, we did not find big influence, because in this experiment, we did not consider node mobility.

2) Evaluation of Link Quality Extension: In Fig. 10, we show the experimental results for throughput of MV1 (1 relay node is moving). The horizontal axis shows the source node id and destination node id. The vertical axis shows the throughput (Kbps). As shown in Fig. 10, if we use TCP data flow, we have more overhead so the throughput is decreased compared with UDP data flow. Moreover, the throughputs drop about $50 \%$ after the third hop.

In Fig. 11, we show the experimental results for throughput of MV3 (two mobile nodes). When we use UDP data flow with different protocols or different LQWS, the throughput results are not affected. On the other hand, when we use TCP data flow, the experimental results are affected by LQWS. If we use small LQWS, then we have high throughput, so the $\widehat{\mathrm{RTT}}$ is decreased compared with LQWS100. From experimental results, we found that for OLSR if we use TCP data flow, we got better results when the LQWS value was 10 .

3) Comparison for Different Mobility Models: In order to show the range of variability of the data, we present the measurement data according to the model types, as shown in Table IV. The average throughput (Kbps) is computed at the receiver. As can be seen from the table, when source node is moving, we found that the average throughput was decreased. Especially, for MV5 model, the throughput is decreased much more. This is because of the effect of the relay node movement and it was caused by routing loops.

In all previous experiments, the experimental time was 10 s. From Figs. 12 to 15, we changed the experimental time to 150 s. In Fig. 12, the horizontal axis shows the experimental time (s). The vertical axis shows the throughput (Kbps), which is computed at the receiver. As shown in Fig. 12(a), we can see a stable constant bit rate flow between node 1 and node 2 for each experimental model. But in Fig. 12(b), there are some 


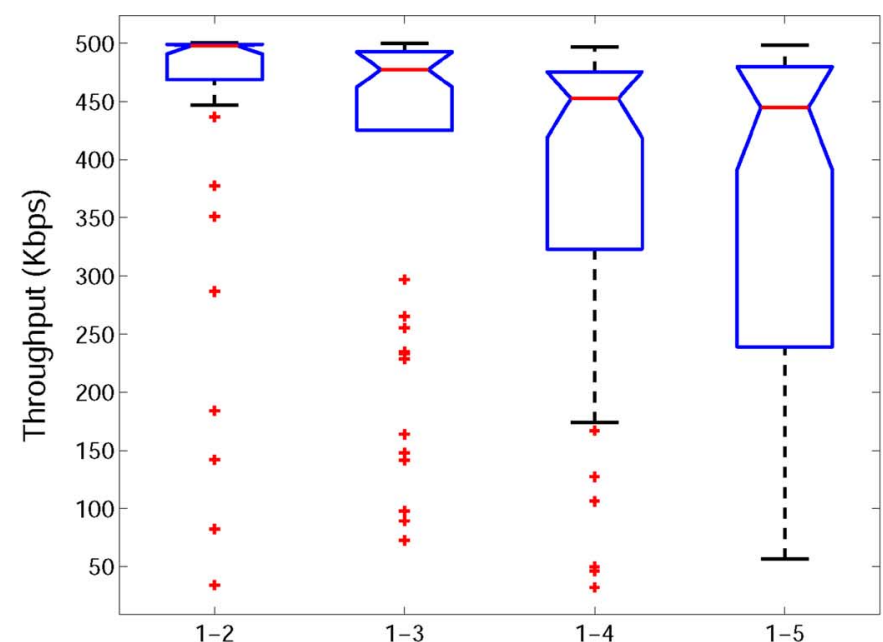

(a)

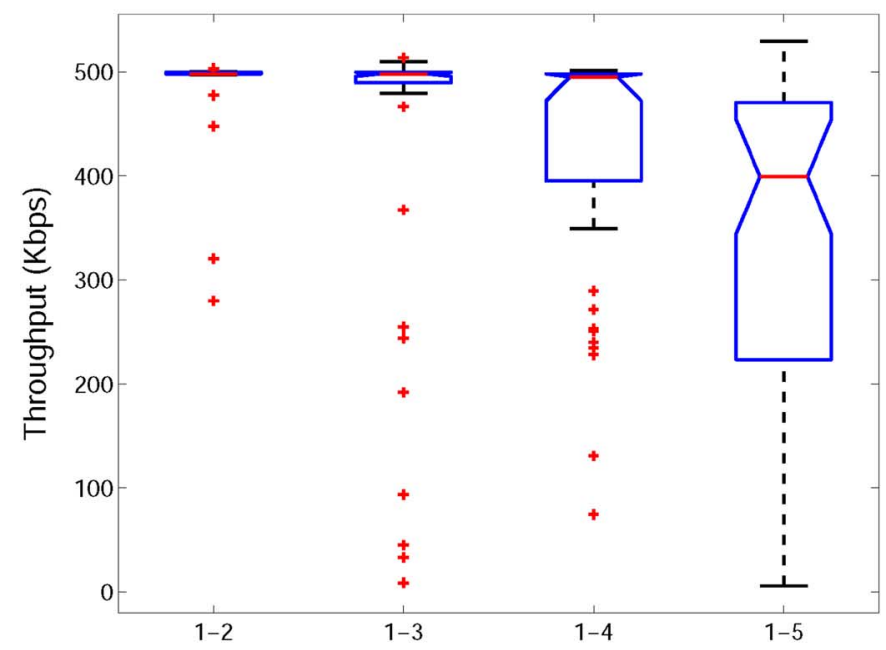

(c)

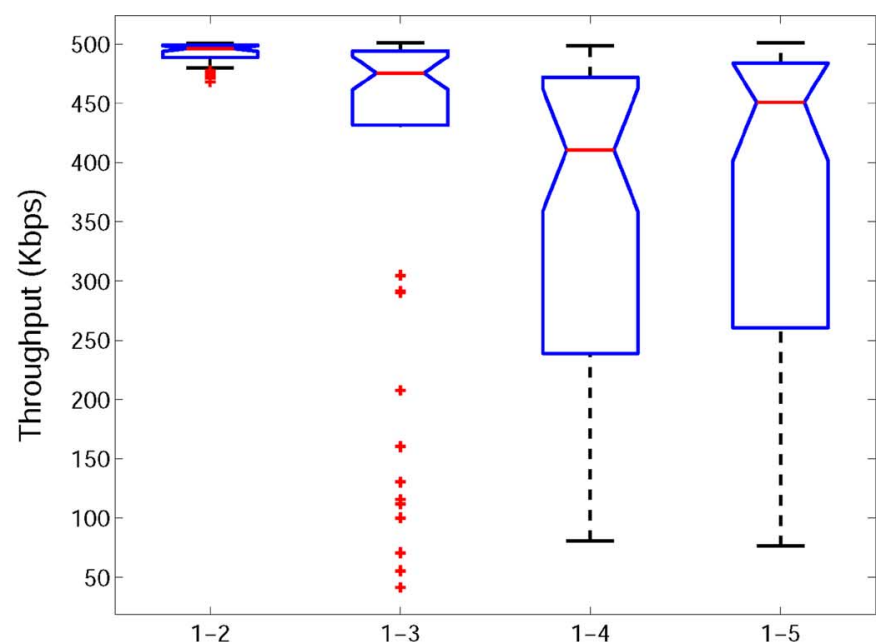

(b)

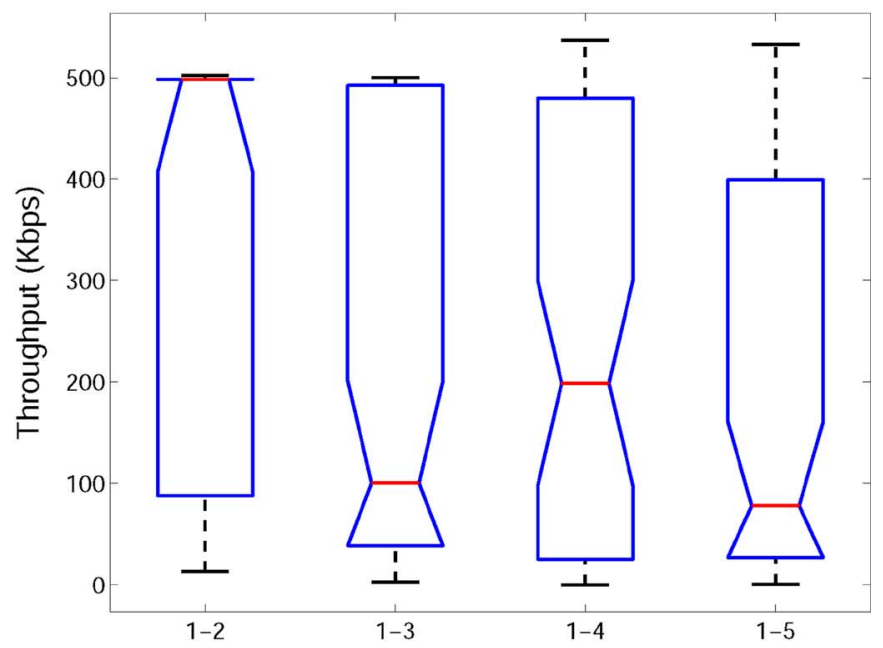

(d)

Fig. 11. Throughput results for MV3. (a) UDP, LQWS10; (b) UDP, LQWS100; (c) TCP, LQWS10; (d) TCP, LQWS100.

TABLE IV

COMPARISON OF AVERAGE THROUGHPUT

\begin{tabular}{c|c|c|c|c}
\hline \hline \multirow{2}{*}{ Model } & \multicolumn{4}{|c}{ Source and destination node id } \\
\cline { 2 - 5 } & $1 \rightarrow 2$ & $1 \rightarrow 3$ & $1 \rightarrow 4$ & $1 \rightarrow 5$ \\
\hline \hline STA, UDP & 499.712 & 480.584 & 499.712 & 499.243 \\
MV1, UDP & 497.443 & 483.567 & 430.656 & 430.656 \\
MV2, UDP & 498.515 & 491.759 & 498.433 & 498.629 \\
MV3, UDP & 498.235 & 447.568 & 455.829 & 408.252 \\
MV4, UDP & 498.863 & 497.569 & 461.049 & 420.674 \\
MV5, UDP & 499.157 & 433.847 & 475.537 & 212.075 \\
MV6, UDP & 499.065 & 461.208 & 493.422 & 438.478 \\
MV7, UDP & & 484.371 & 478.485 & 414.901 \\
\hline \hline
\end{tabular}

oscillations in MV3, MV5 and MV6 models. This is because of movement of node 3. Moreover, the number of packet loss increases after node $1 \rightarrow 4$ as shown in Fig. 13. It seems that the topology can be very dynamic in MV3 and MV5 models. We experienced a lot of oscillation in MV5 model as shown in Fig. 13(d). A direct symmetric link exists between nodes 1 and 4 , and OLSR chooses correctly the 2-hops route, i.e., 1-2-4 or 1-3-4. In this case, the hop-count threshold is 3 , and, again, we find high variability for the connection 1-4, which incurs in the longest route, e.g., 1-2-3-4.
In Figs. 14 and 15, we show the throughput and packet loss during node join and leave operations. In Fig. 14(b) is shown that even node 2 is leaving between 50 and $100 \mathrm{~s}$, there is not a big effect on the throughput of link between node 1 and node 3 (because node 3 is within transmission distance of node 1). However, we can see a big effect on the UDP throughput for node 4 and node 5 [see Fig. 14(c) and (d)]. In Fig. 15, we can see that in MV7 model the packet loss is higher compared with STA model and MV1 model.

From these results, we conclude that the UDP data flow is affected more in MV5 compared with MV6. We found that OLSR protocol has a good performance when the source node is moving. However, the performance is not good when the relay nodes are moving. Therefore, the OLSR protocol needs to be equipped with more realistic topology control mechanism.

\section{CONCLUSION}

In this paper, we presented the implementation, experiences and lessons learned of our tesbed for Ad-hoc networks and MANETs. We used OLSR protocol for real experimental evaluation. We considered three parameters for performance 


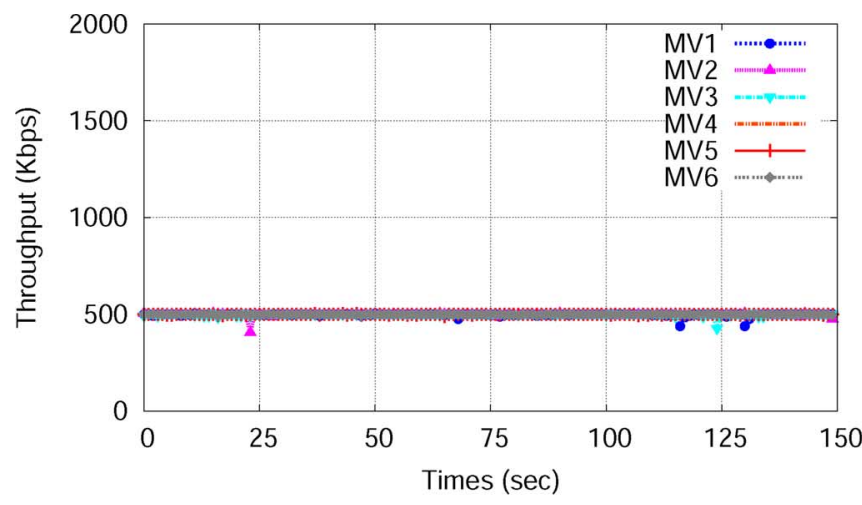

(a)

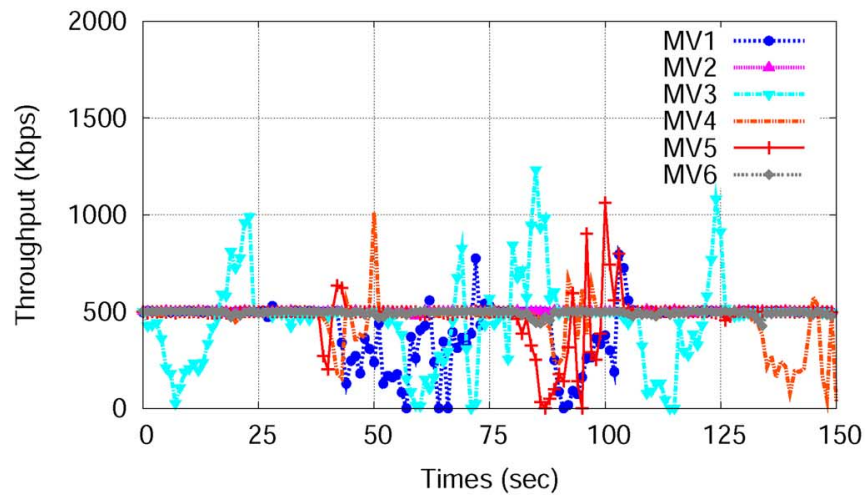

(c)

Fig. 12. UDP throughput results for moving models. (a) UDP, $1 \rightarrow 2$; (b) UDP, $1 \rightarrow 3$; (c) UDP, $1 \rightarrow 4$; (d) UDP, $1 \rightarrow 5$

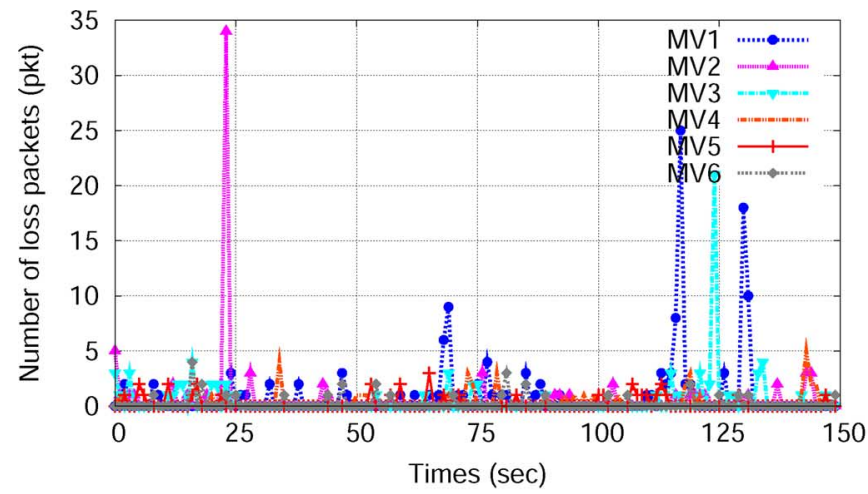

(a)

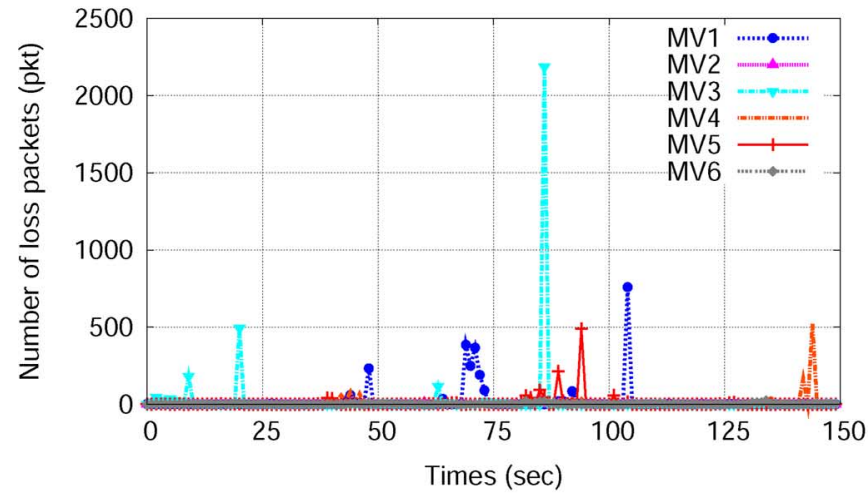

(c)

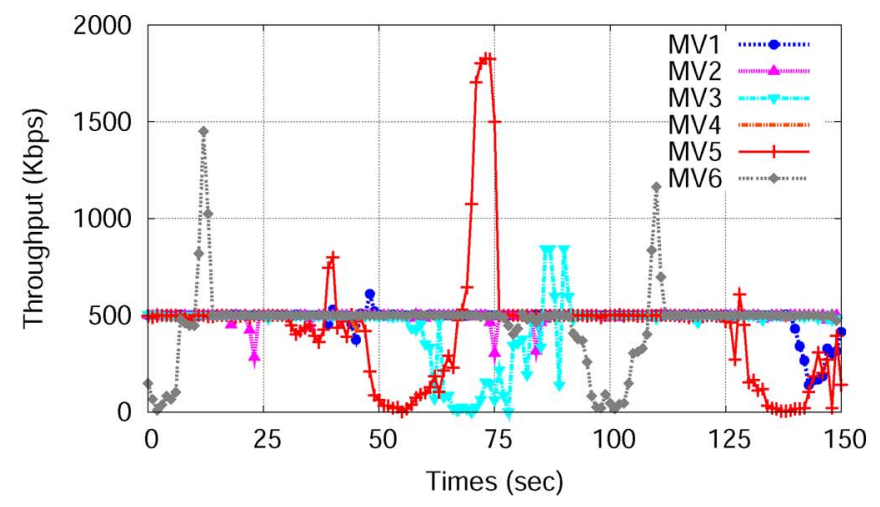

(b)

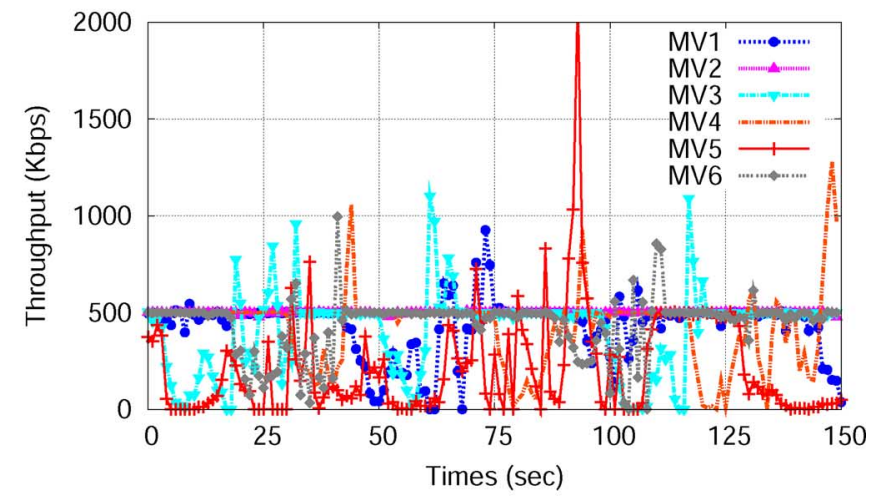

(d)

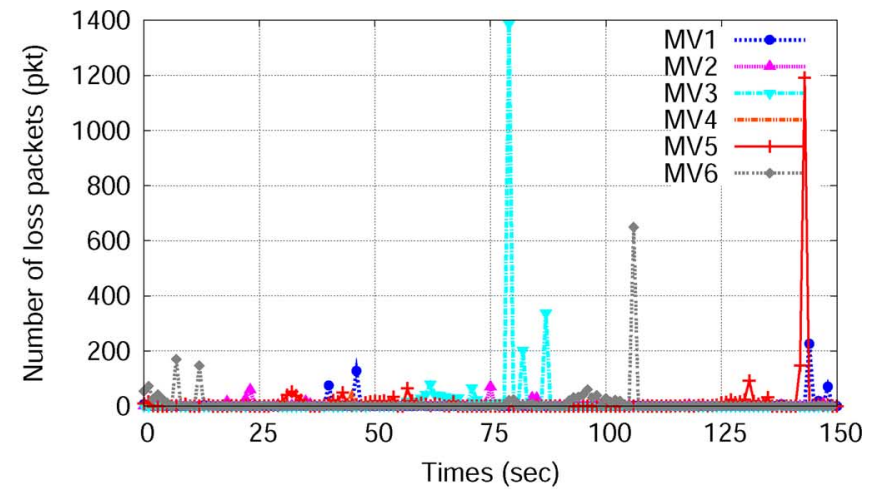

(b)

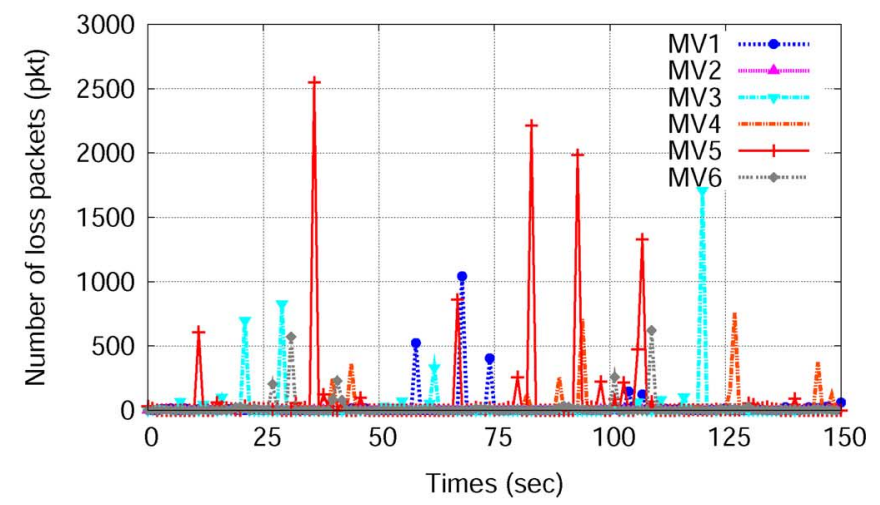

(d)

Fig. 13. Packet loss results for moving models. (a) UDP, $1 \rightarrow 2$; (b) UDP, $1 \rightarrow 3$; (c) UDP, $1 \rightarrow 4$; (d) UDP, $1 \rightarrow 5$.

evaluation: throughput, RTT and packet loss. In our experiments, we considered eight models: STA, MV1, MV2, MV3, MV4, MV5, MV6, and MV7.
From the experiments, we found the following results. We proved that while some of the OLSR's problems can be solved, for instance the routing loop, this protocol still have the self-in- 


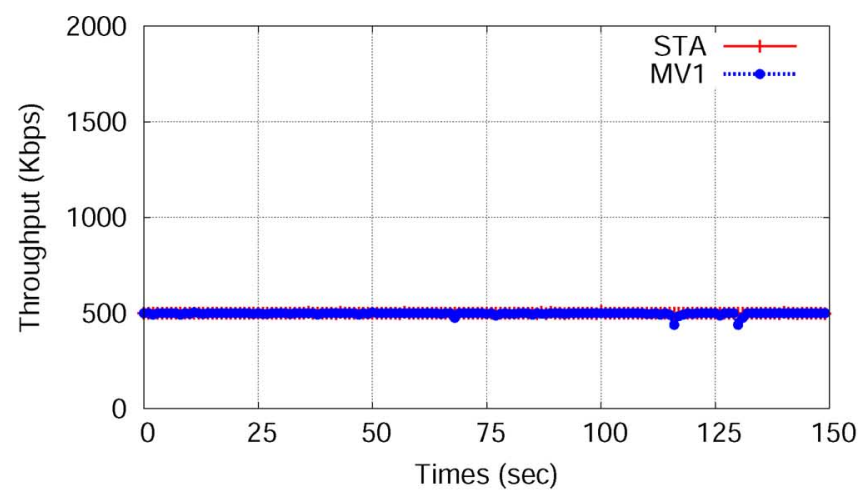

(a)

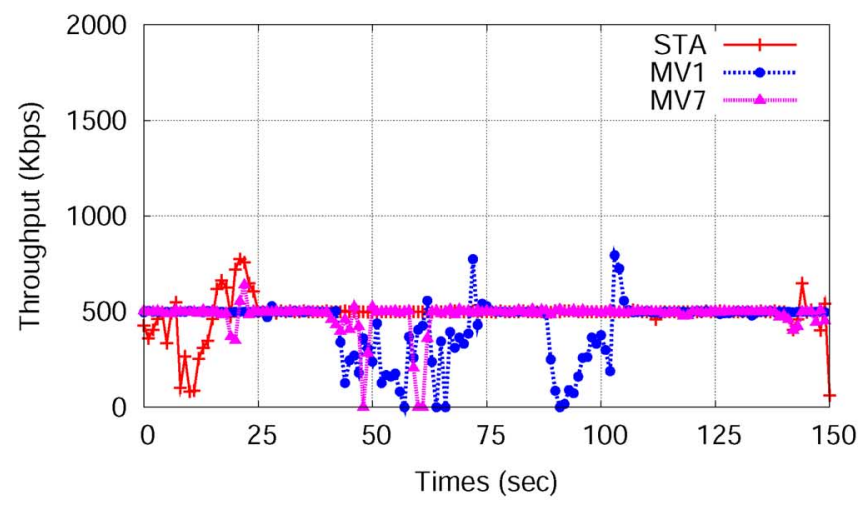

(c)

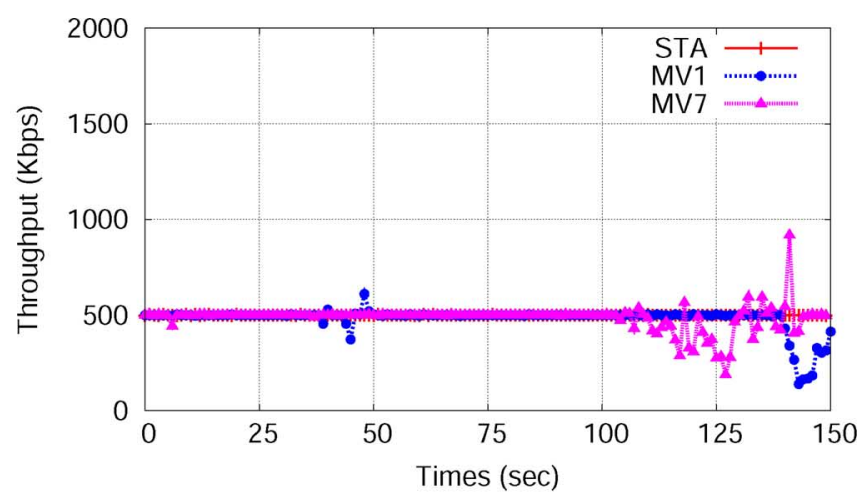

(b)

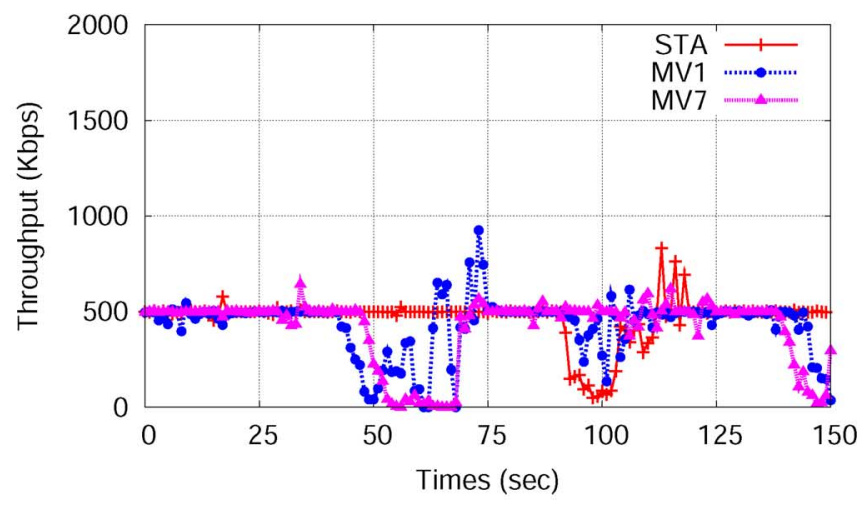

(d)

Fig. 14. Comparison of UDP throughput for STA, MV1 and MV7. (a) UDP, $1 \rightarrow 2$; (b) UDP, $1 \rightarrow 3$; (c) UDP, $1 \rightarrow 4$; (d) UDP, $1 \rightarrow 5$.

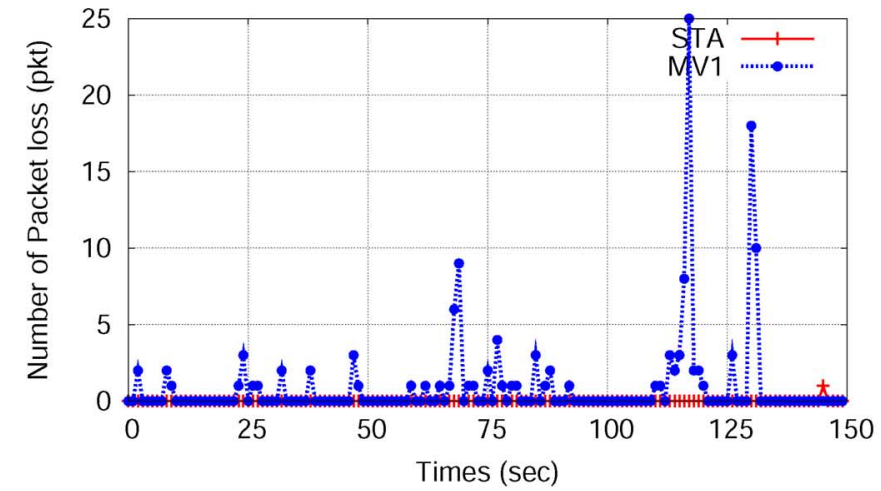

(a)

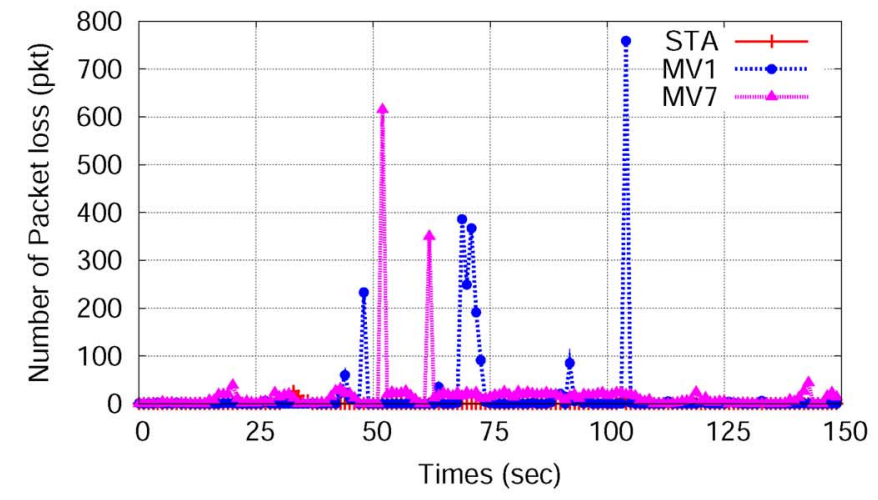

(c)

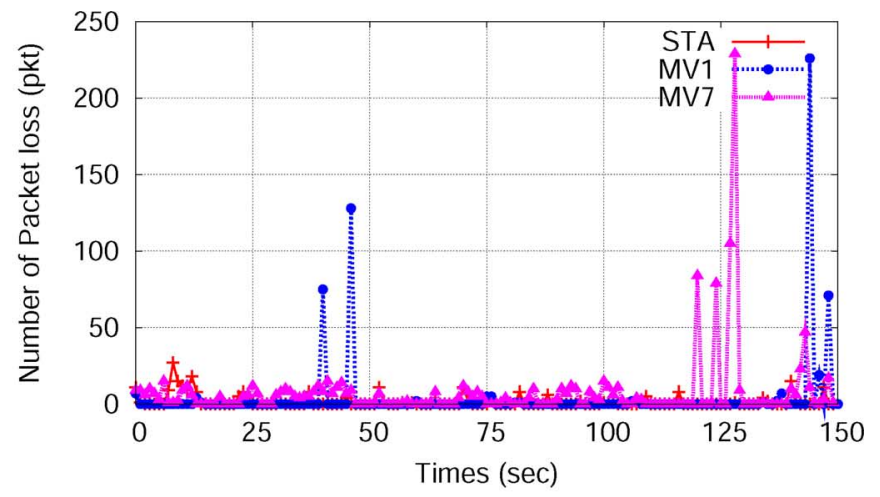

(b)

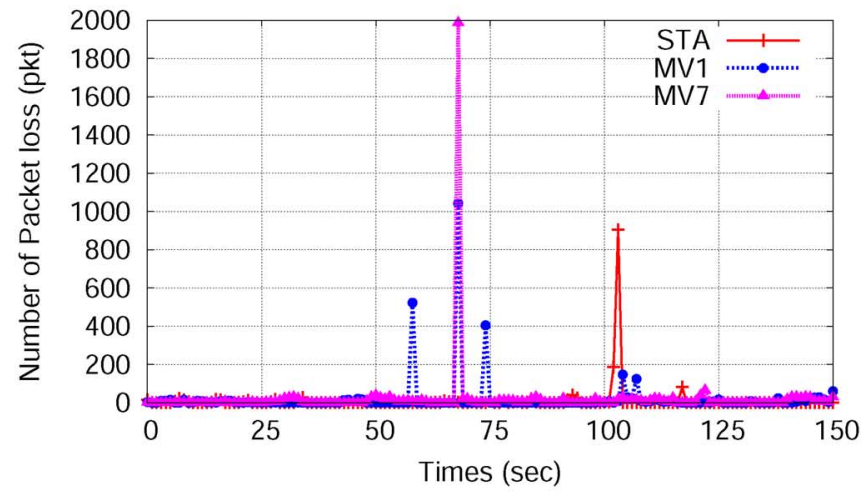

(d)

Fig. 15. Comparison of packet loss for STA, MV1 and MV7. (a) UDP, $1 \rightarrow 2$; (b) UDP, $1 \rightarrow 3$; (c) UDP, $1 \rightarrow 4$; (d) UDP, $1 \rightarrow 5$.

terference problem. There is an intricate interdependence between MAC layer and routing layer, which can lead the experimenter to misunderstand the results of the experiments. For example, the horizon is not caused only by IEEE 802.11 Distributed Coordination Function (DCF), but also by the routing protocol. We carried out the experiments considering stationary 
nodes of an ad-hoc network and the node mobility of MANETs. We found that throughput of TCP were improved by reducing Link Quality Window Size (LQWS), but there were packet loss because of experimental environment and traffic interference. For TCP data flow, we got better results when the LQWS value was 10 . Moreover, we found that the node join and leave operations increase the packet loss. The OLSR protocol has a good performance when the source node is moving. However, the performance is not good when the relay nodes are moving. There are some oscillations in MV3, MV5 and MV6 models. This is because of node movement.

Our experimental results will shed light for the real implementation of Ad-hoc and MANETs.

The experiments where performed using a single flow through the network. In the future, we would like to consider the effect of multiple flows on the routing and increase the number of nodes. Moreover, we would like to extend our testbed.

\section{ACKNOWLEDGMENT}

The authors would like to thank JSPS for their support.

\section{REFERENCES}

[1] H. Lundgren, E. Nordströ, and C. Tschudin, "Coping with communication gray zones in IEEE $802.11 \mathrm{~b}$ based ad hoc networks," in WOWMOM'02: Proc. 5th ACM Int. Workshop on Wireless Mobile Multimedia, 2002, pp. 49-55.

[2] W. Kiess and M. Mauve, "A survey on real-world implementations of mobile ad-hoc networks," Ad Hoc Networks, vol. 5, no. 3, pp. 324-339, 2007.

[3] A. Tønnesen, OLSRd: Implementation Code of the OLSR [Online]. Available: http://www.olsr.org/

[4] D. A. Maltz, J. Broch, and D. B. Johnson, "Lessons from a full-scale multihop wireless ad hoc network testbed," IEEE Personal Commun., vol. 8, no. 1, pp. 8-15, Feb. 2001.

[5] R. S. Gray, D. Kotz, C. Newport, N. Dubrovsky, A. Fiske, J. Liu, C. Masone, S. McGrath, and Y. Yuan, "Outdoor experimental comparison of four ad hoc routing algorithms," in Proc. MSWiM'04, 2004, pp. 220-229.

[6] G. Anastasi, E. Borgia, M. Conti, and E. Gregori, "IEEE 802.11b ad hoc networks: Performance measurements," Cluster Comput., vol. 8, no. 2-3, pp. 135-145, 2005.

[7] V. Kawadia and P. R. Kumar, "Experimental investigations into tcp performance over wireless multihop networks," in Proc. E-WIND-2005, 2005, pp. 29-34.

[8] T. Clausen, G. Hansen, L. Christensen, and G. Behrmann, "The optimized link state routing protocol, evaluation through experiments and simulation," in Proc. IEEE Symp. Wireless Personal Mobile Communications, Sep. 2001.

[9] D. Johnson and G. Hancke, "Comparison of two routing metrics in OLSR on a grid based mesh network," Ad Hoc Networks 2008, May 4, 2008.

[10] G. De Marco, M. Ikeda, T. Yang, and L. Barolli, "Experimental performance evaluation of a pro-active ad-hoc routing protocol in out- and indoor scenarios," in Proc. AINA-2007, Niagara Falls, ON, Canada, May 2007, pp. 7-14.

[11] T. Clausen and P. Jacquet, "Optimized link state routing protocol (OLSR)," RFC 3626 (Experimental), 2003.

[12] D. S. J. D. Couto, D. Aguayo, J. Bicket, and R. Morris, "A high-throughput path metric for multi-hop wireless routing," in Proc. MobiCom-2003, 2003, pp. 134-146.

[13] Rt2x00 Project [Online]. Available: http://rt2x00.serialmonkey.com/

[14] A. Botta, A. Dainotti, and A. Pescape, "Multi-protocol and multi-platform traffic generation and measurement," in INFOCOM 2007 DEMO Session, May 2007.
[15] L. Barolli, M. Ikeda, G. De Marco, A. Durresi, and X. Fatos, "Performance analysis of OLSR and BATMAN protocols considering link quality parameter," in Proc. AINA 2009, Bradford, U.K., May 2009, pp. 307-314.

[16] M. Ikeda, L. Barolli, G. De Marco, T. Yang, and A. Durresi, "Experimental and simulation evaluation of OLSR protocol for mobile ad-hoc networks," in Proc. NBiS-2008, Turin, Italy, Sep. 2008, pp. 111-121.

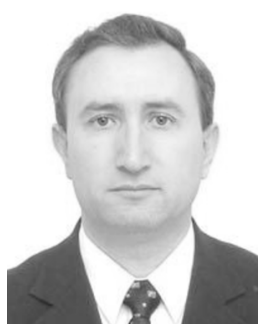

Leonard Barolli (M'01) is a Full Professor, Department of Information and Communication Engineering, Fukuoka Institute of Technology (FIT), Japan. He has published about 300 papers in referred journals, books and international conference proceedings. His research interests include network traffic control, fuzzy control, genetic algorithms, agent-based systems, ad-hoc networks and sensor networks.

Dr. Barolli has served as a Guest Editor for many international journals. He has been a PC Member, PC Chair and General Chair of many international conferences. He is the Steering Committee Chair of CISIS International Conference and is serving as Steering Committee Co-Chair of IEEE AINA, NBiS, BWCCA and 3PGCIC International Conferences. He has won many Awards for his scientific work and has received many research funds. He is a member of SOFT and IPSJ.

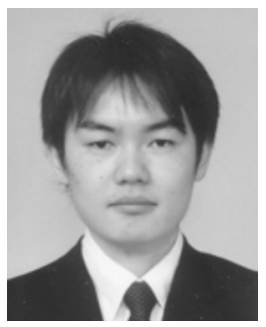

Makoto Ikeda (M'09) received the B.E., M.E., and $\mathrm{Ph} . \mathrm{D}$. degrees in information and communication engineering from Fukuoka Institute of Technology (FIT), Japan, in 2005, 2007, and 2010, respectively.

He is an Assistant Research Fellow at Seikei University, Japan. He has published about 30 research papers in journals and international conference proceedings. His research interests include wireless networks, mobile computing, high-speed networks, P2P systems, ad-hoc networks and sensor networks.

Dr. Ikeda won the Best Paper Award at NBiS-2008 International Conference and has been a PC Member and Web Administrator for some International Conferences. He is a member of the ACM, IPSJ and IEICE.

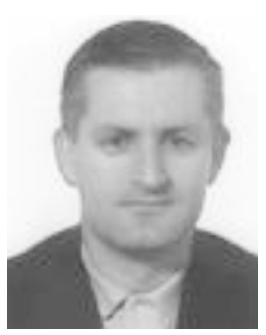

Fatos Xhafa (M'09) is currently a Visiting Professor with the Department of Computer Science and Information Systems, Birkbeck, University of London. $\mathrm{He}$ is Associate Professor at the Technical University of Catalonia, Spain. His main research interests include parallel and distributed algorithms, combinatorial optimization, Grid and P2P computing. He has widely published in peer-reviewed international journals, books and conference proceedings of the research area.

Dr. Xhafa serves the editorial board of nine peerreviewed international journals and has also guest co-edited in several international journals. He has served and is currently serving as PC Co-Chair/General Co-Chair of several international conferences and workshops.

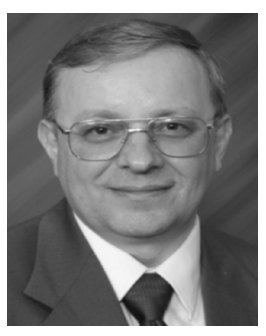

Arjan Durresi (M'98-SM'02) is an Associate Professor in the Department of Computer and Information Science at Indiana University-Purdue University at Indianapolis. he has authored more than 70 journal papers and more than 100 conference papers. He has organized many international workshops. His current research interests include network architectures, heterogeneous wireless networks, security, QoS routing protocols, traffic management, and optical and satellite networks.

Dr. Durresi has delivered several keynote speeches at international conferences, including IEEE AINA 2007 and NBiS 2009. He is a recipient of several paper awards in international conferences. 